Short communication

\title{
Diffraction diamond grating formed by silver-ion mask implantation
}

\author{
A.L. Stepanov ${ }^{\mathrm{a}, *}$, V.I. Nuzhdin ${ }^{\mathrm{a}}$, N.M. Kazuchits ${ }^{\mathrm{b}}$, V.F. Valeev ${ }^{\mathrm{a}}$ \\ ${ }^{a}$ Kazan Physical-Technical Institute, Russian Academy of Sciences, 420029, Kazan, Russia \\ ${ }^{\mathrm{b}}$ Belarus State University, 220030, Minsk, Belarus
}

A R T I C L E I N F O

Keywords:

Diamond

Ion implantation

Silver nanoparticles

Diffraction grating

The paper shows a novel method for fabrication of a diffraction grating on the surface of synthetic diamond by implantation with silver ions through a mask. It was found for the first time that, in the process of ion implantation, silver nanoparticle formation and ion etching of the diamond surface are realized in the non-masked areas of diamond in near-surface region. These structural transformations of the diamond surface were controlled by optical, confocal, electron, and atomic force microscopy. Raman spectroscopy was also used to identify the absence of graphite formation during ion implantation of diamond. The operating efficiency of a diamond optical diffraction element was demonstrated when it was probed by radiation with a semiconductor laser at a wavelength of $527 \mathrm{~nm}$.

In practice modern integrated optics requires to apply new specific materials, as well as the development of special technologies for the manufacture of working components and devices. One of direction of integrated optics is based on the use of optical components created from diamond that is called diamond integrated optics [1]. The use of diamond as optical materials is effective due to its unique radiation durability and high thermal conductivity. Diamond optical elements, вуь\#тыекфе\#тп а wide transparency from 0.2 to more than $20 \mu \mathrm{m}$, could work with sharp temperature drops and in aggressive chemical environments. Currently, diamond elements are applied to make various diffractive optical elements (DOEs): gratings, kinoforms, focusators, proofreaders, etc. [2,3]. Diamond DOEs could convert optical radiation from a high-power $\mathrm{CO}_{2}$ laser with a power density of up to $20 \mathrm{~kW} / \mathrm{cm}^{2}$ [4], to create Bragg superlattice for obtaining individual photoluminescence of diamond color centers [5], as well as in X-ray optics for control of radiation fluxes, for example, using diamond Bragg mirrors (reflecting diffraction gratings) with a reflection coefficient of $100 \%[6,7]$ and so on.
For the fabrication of periodic DOEs on the surface of different optical materials, various technological methods are used, such as electron lithography [8], deposition of colloidal microspheres [9], twophoton polymerization by femtosecond laser pulses [10], etc. Special interest for the manufacture of DOE is a technology based on ion implantation through a surface mask. Recently, this approach was successfully implemented to create DOE by implantation with various ions through masks on such optically transparent materials as silica [11,12] and polymethylmethacrylate [13]. Moreover, the implanted regions of periodic elements were contained ion-synthesized plasmonic silver or copper nanoparticles. Collective excitation of conduction electrons in these nanoparticles (surface plasmon resonance - SPR) under the excitation of an electromagnetic light wave and the resulting resonant amplification of the local field significantly modify the dielectric constants of effective media at specific light frequencies, which leads to an increased phase contrast in the DOEs [14].

To obtain periodic DOEs on diamond substrates, such technological methods as exposure by powerful pulses from an excimer laser [3], plasma etching in a transport gas stream [2], etc. are applied. It was also suggested to creating diamond DOEs [15] by high-dose implantation of single-crystal diamond plates with boron ions through surface masks.

The aim of this study was to develop the proposed method for fabrication of DOEs with plasmonic nanoparticles on a diamond substrate by ion implantation through masks using silver ions. The silver nanoparticles synthesized in the DOE structure could allow using their plasmon properties to control the choice of optical constants in the DOE structure, similarly to how it was previously realized on silicate glasses and polymers [11-13]. It should be noted that attempts to implant diamond with ions of noble metals (gold and silver) were made earlier

\footnotetext{
* Corresponding author.

E-mail address: aanstep@gmail.com (A.L. Stepanov).
} 
[16-18], but the creation of DOEs in the mentioned experiments was not implemented.

To form diamond DOE, the polished surface of an artificial optically transparent colorless diamond was implanted by silver ions with an energy of $E=30 \mathrm{keV}$, an a dose of $D=1.3 \cdot 10^{18} \mathrm{ion} / \mathrm{cm}^{2}$ and a current ion density of $J=20 \mu \mathrm{A} / \mathrm{cm}^{2}$ with the ILU-3 accelerator at room temperature of the substrate. The vacuum level in an ion accelerate chamber during ion implantation was $10^{-5}$ Torr. Nickel mesh with a size of square cells of $40 \mu \mathrm{m}$ as a surface mask was used. The local morphology and surface structure of the implanted diamond were examined by scanning electron microscopy (SEM) with Merlin SEM microscope (Carl Zeiss) and atomic force microscopy (AFM) in a semicontact mode using Dimension FastScan AFM microscope (Brucker). The optical characterization of the DOE was carried out with optical polarization microscope Polar-1 (Micromed) in the reflection mode and with an confocal microscope LSM 780 (Carl Zeiss), as well as with the measurement of Raman spectra. The analysis of optical diffraction patterns from the DOE was demonstrated when it was probed with a semiconductor laser at a wavelength of $527 \mathrm{~nm}$.

Modeling of the concentration depth profiles of the implanted silver distribution for accelerating energy of $30 \mathrm{keV}$ into diamond using the SRIM-2013 algorithm showed that silver atoms accumulate in the surface layer of diamond $\sim 25 \mathrm{~nm}$ thick without penetrating into the depth of the sample.

Fig. 1 shows the images of a grating fabricated on the surface of a diamond by implantation with silver ions through a surface mask, observed in optical (Fig. 1a) and by SEM (Fig. 1b) microscopes. As seen from the images, the microstructure consists of periodic dark square cells belonging to implanted areas of the sample surface separated by walls (bright region) of non-implanted diamond. The size of the implanted areas of $40 \times 40 \mu \mathrm{m}$ corresponds to the size of the cells used mask. The images also show that the implanted cells in the diamond structure are below the surface level of the virgin material, indicating the sputtering of the diamond with silver ions. The observed triangular surface structures were existed in the diamond before implantation which are defects (as etching pits) formed during the synthesis of diamond and the cutting of the bulk sample to thin substrates.

SEM images with various scales of the diamond surface in the area of a single grating cell implanted with silver ions are shown in Fig. 2a and b. It could be seen in the structure of the smooth surface of a implanted diamond bright spherical spots. These spots correspond to silver nanoparticles similar to nanoparticle observation in the work [18]. The formation of metal nanoparticles at a given $D$ value is explained by the accumulation of silver atoms in amounts exceeding a solubility limit in implanted matrix layer $>10^{16} \mathrm{~cm}^{-3}$ similar as it were experimentally observed for metal-ion implantation of silica or oxide semiconductors [19,20].

Fig. 3a shows an AFM-image of a surface fragment of an implanted diamond grating cell near the unirradiated areas (between the wall) which were closed during implantation by a mask. This AFM image also confirms that after silver ion implantation the unmasked areas of the sample were subjected to ion sputtering, and as a result of which gratings structure (square periodic holes) were formed. In Fig. 3b an AFM image is shown with higher magnifications in the region of the gratings cell, in which the synthesized silver nanoparticles are present, as it was also observed in SEM images (Fig. 2) or by AFM analysis in the work [18].

The formation of a grating on the surface of a diamond during silver ions implantation through a surface mask is confirmed by observing the sample in an optical confocal microscope (Fig. 4). As a probe signal, a semiconductor laser at a wavelength of $488 \mathrm{~nm}$ was used, and the optical image of the sample was recorded in the spectral region 508-526 nm through cut-off filters. The impact of the study at a wavelength of $488 \mathrm{~nm}$ leads to the excitation of luminescence of diamond light in the visible green spectrum. Fig. 4 shows an optical 3D-image obtained as a result of laser irradiation of a diamond for the surface areas covered during the implantation by the mask. The areas of implanted diamond (square cells) practically do not luminesce and therefore demonstrate dark color in the figure.

Raman spectra upon excitation with an argon laser at a wavelength of $514 \mathrm{~nm}$ does not indicate the formation of any graphitized regions in diamond, as was observed, for example, during diamond implantation under similar conditions with boron ion [15]. The explanation for such observation could be given by the effect of intense sputtering of a diamond surface with by heavy silver ions, in which all graphitized fragments of diamonds are removed almost immediately after ion implantation was started. In this case, the surface depth profile is formed, but the graphitized elements of the sample are not preserved in grating structure.

The implantation of diamond with silver ions leads to a change in the phase composition in general due to the formation of silver nanoparticles. The ion implantation of diamond through the surface mask fabricate a microstructure with a periodically variable distribution of the optical constants of the composite material, i.e. between the walls of the diamond grating and cells filled by silver nanoparticle. Therefore, a periodic microstructure formed on diamond with areas containing metal nanoparticles could be used in practice as a two-dimensional photonic crystal or DOEs.

As an illustration in Fig. 5, it shows a diffraction image recorded when a diamond grating is probed by a semiconductor green laser at the wavelength of $527 \mathrm{~nm}$. Obviously, by manipulating the parameters and conditions of ion implantation, it is possible to thereby change the effective refractive index of individual areas in the diamond DOEs, i.e. manage optical and diffraction characteristics.

Thus, in the present work, low-energy high-dose implantation of diamond with silver ions through a surface mask was implemented for the first time and a new method for obtaining DOE was suggested. As a result, a diffraction grating microstructure was obtained on the surface
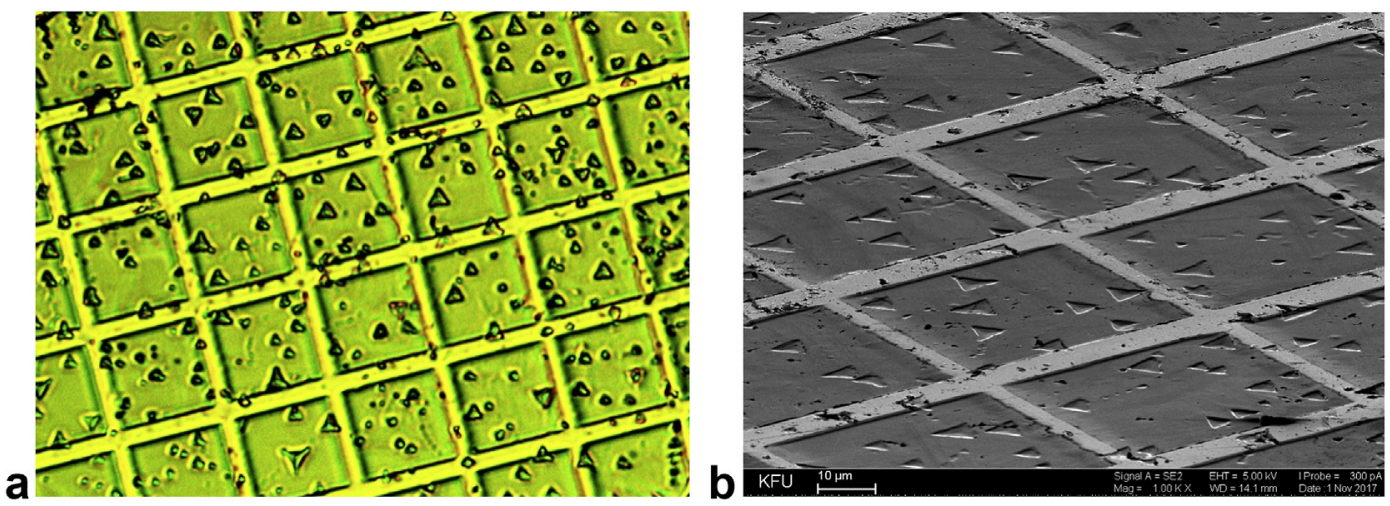

Fig. 1. Images of diamond implanted through a mask with silver ions, observed in optical (a) and electron (b) microscopes. 


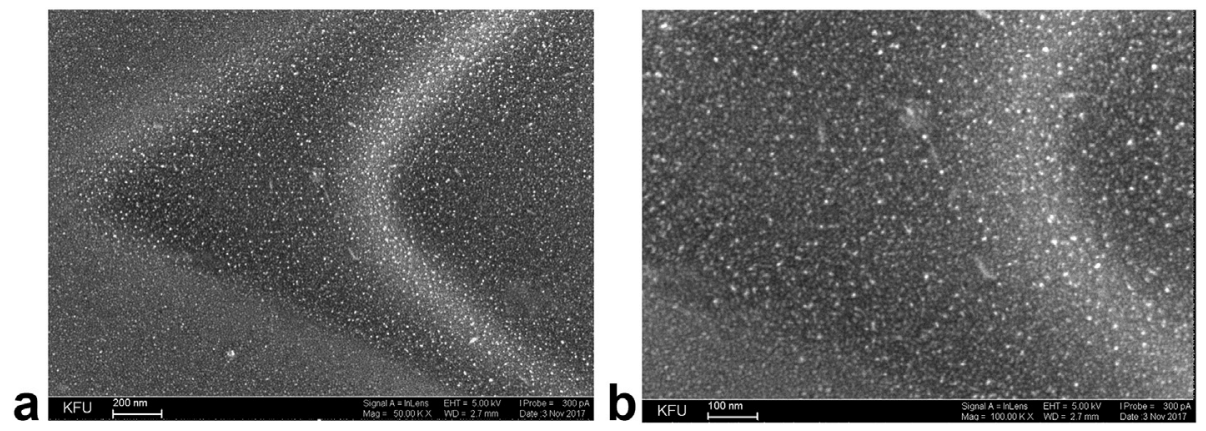

Fig. 2. SEM images of a diamond surface implanted with silver ions in a region of periodic formed cell.

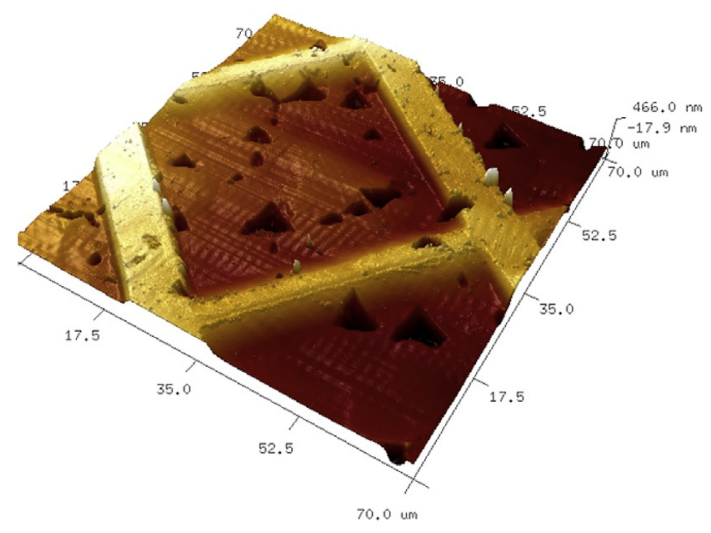

a

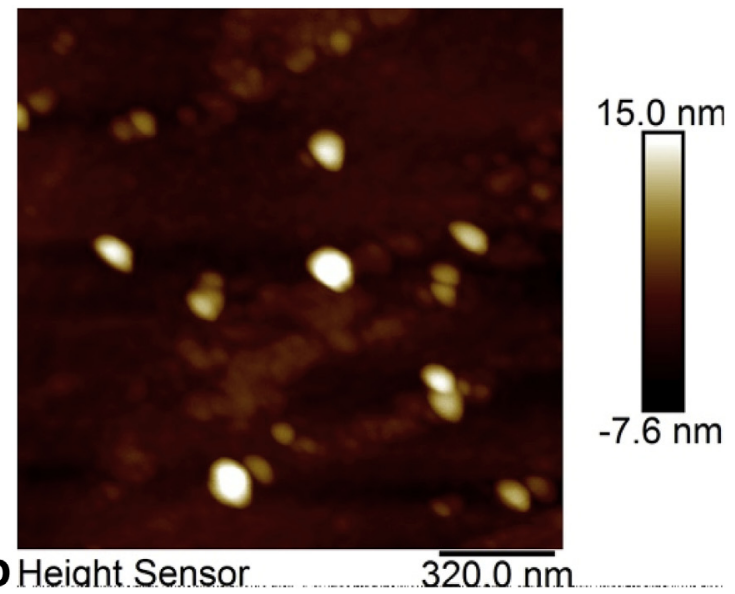

b Height Sensor

$3 \overline{20.0 \mathrm{~nm}}$

Fig. 3. An AFM image of the surface of a diamond diffraction grating fragment: (a) in the areas of the non-irradiated diamond septum (the bright part of the pattern) and implanted cells (the dark parts of the picture), as well as the grating regions with silver nanoparticles.

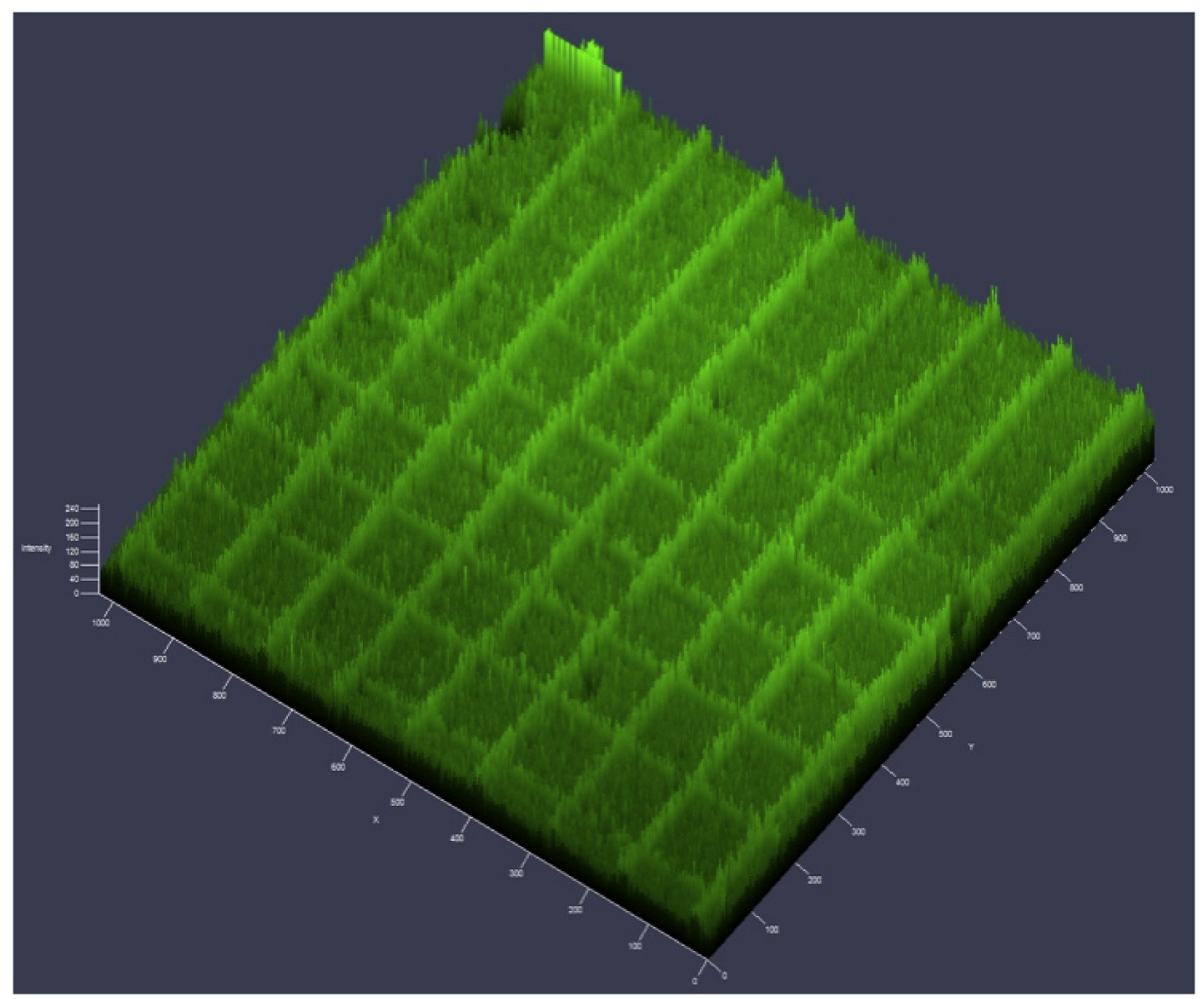

Fig. 4. 3D-image of a diamond grating fragment observed in an optical confocal microscope. 


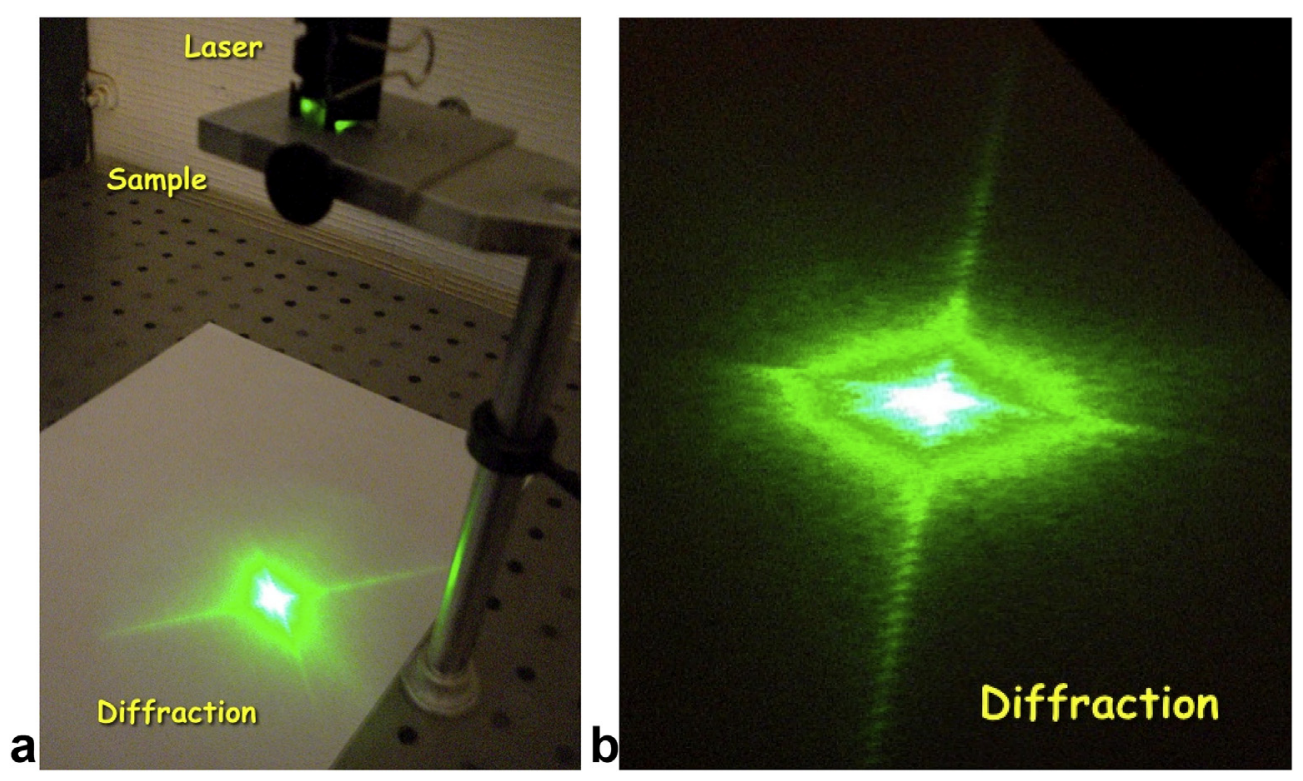

Fig. 5. Image of a diffraction scatter pattern on the screen, obtained from a diamond surface lattice when probing with a green laser in transmission geometry. (For interpretation of the references to color in this figure legend, the reader is referred to the Web version of this article.)

of the diamond, the phase contrast is provided by periodic areas of diamond with plasmonic silver nanoparticles. The main practical application of the proposed method of forming DOE is the development of promising technology and the creation of new effective elements of diamond optics.

\section{Acknowledgement}

Authors thanks N.V. Kurbatova form Kazan Physical-Technical Institute for Raman spectral measurements and V.V. Vorobev and Y.N. Osin from Kazan Federal University for help with some microscopy observation.

\section{References}

[1] A.D. Greentree, B.A. Fairchild, F.M. Hossain, S. Prawer, Mater. Today 11 (2008) 22-31.

[2] M. Karlsson, F. Nikolajeff, Optic Express 11 (2003) 502-507.

[3] T.V. Kononenko, V.V. Kononenko, V.I. Konov, S.M. Pimenov, S.V. Garnov, A.V. Tishchenko, A.M. Prokhorov, A.V. Khomich, Appl. Phys. A 68 (1999) 99-102.

[4] V.V. Kononenko, V.I. Konov, S.M. Pimenov, A.M. Prokhorov, V.S. Pavelev, V.A. Soifer, Quant. Electron. 29 (1999) 9-10.

[5] V.A. Kukushkin, Appl. Phys. A 123 (2017) 663-1 - 663-7.
[6] M. Makita, P. Karvinen, V.A. Guzenko, N. Kujala, P. Vagovic, C. David, Microelectron. Eng. 176 (2017) 75-78.

[7] Y. Shvydko, S. Stoupin, V. Blank, S. Terentyev, Nat. Photon. 5 (2011) 539-542.

[8] A.B. Evlyukhin, S.I. Bozhevolnyi, A.L. Stepanov, J.R. Krenn, Appl. Phys. A 84 (2006) 29-34.

[9] M.S. Ashurov, T.A. Kazakova, A.L. Stepanov, S.O. Klimonsky, Appl. Phys. A 122 (2016) 1954-1 -1054-8.

[10] A.L. Stepanov, R. Kiyan, A. Ovsianikov, V.I. Nuzhdin, V.F. Valeev, B.N. Chichkov, Appl. Phys. A 108 (2012) 375-378.

[11] A.L. Stepanov, M.F. Galyatdinov, A.B. Evlyukhin, V.I. Nizhdin, V.F. Valeev, Y.N. Osin, R. Kiyan, T.S. Kavetskyy, B.N. Chichkov, Appl. Phys. A 111 (2013) 261-264.

[12] J. Wang, X. Mu, G. Wang, C. Liu, Opt. Mater. 73 (2017) 466-472.

[13] M.F. Galyautdinov, V.I. Nuzhdin, Y.V. Fattakhov, B.F. Farrakhov, V.F. Valeev, A.L. Stepanov, Tech. Phys. Lett. 42 (2016) 182-185.

[14] U. Kreibig, M. Vollmer, Optical Properties of Metal Clusters, Springer, Berlin, 1995.

[15] A.L. Stepanov, V.I. Nuzhdin, M.F. Galyautdinov, V.F. Valeev, N.V. Kurbatova, V.V. Vorobev, Y.N. Osin, Bull. Russ. Acad. Sci. Phys. 82 (2018) 1047-1051.

[16] K.J. Sankaran, B. Sundaravel, N.H. Tai, I.N. Lin, J. Appl. Phys. 118 (2015) 85306-1 85306-9.

[17] K. Panda, J.J. Hyeok, J.Y. Park, K.J. Sankaran, S. Balakrishnan, I.-N. Lin, Sci. Rep. 7 (2017) 16325-1 -16325-14.

[18] Y. Shen, Y.Q. He, S. Yu, Mater. Lett. 139 (2015) 322-324.

[19] A.L. Stepanov, D.E. Hole, P.D. Townsend, J. Non-Cryst. Solids 244 (1999) 275-279.

[20] A. Cetin, R. Kibar, M. Ayvaclkh, N. Can, C. Buchal, P.D. Townsend, A.L. Stepanov, T. Karah, S. Selvi, Nucl. Instrum. Methods Phys. Res. B 249 (2006) 474-477. 